H I G H L I G H T S

\section{Silencing the silencers}

The production of small interfering RNAs (siRNAs) from doublestranded RNA (dsRNA) precursors provides a mechanism for silencing of specific mRNAs, and is known as RNA interference (RNAi). Now, Li et al. report that such a pathway provides antiviral immunity in that this protection is suppressed by proteins encoded by influenza and vaccinia viruses, implicating RNAi as a natural antiviral response in mammalian cells.

The $\mathrm{B} 2$ protein of nodavirus flock house virus (FHV) was shown to inhibit silencing of viral RNAs in Drosophila cells, as well as prevent the degradation of mRNA specifically targeted by homologous siRNA constructs. Similarly, the B2 protein of a second nodavirus - nodamura virus $(\mathrm{NoV})$ - also inhibited the RNAi antiviral response of Drosophila cells, as did a protein encoded by tombusvirus (a plant pathogen). In addition, NoV infection of mosquito cells also activated an RNAi-mediated antiviral response that was susceptible to $\mathrm{B} 2$ silencing, establishing that this pathway of viral immunity functions in more than one invertebrate species. Taken together, these studies imply conservation of this RNAimediated RNA-silencing antiviral response between the plant and animal kingdoms.

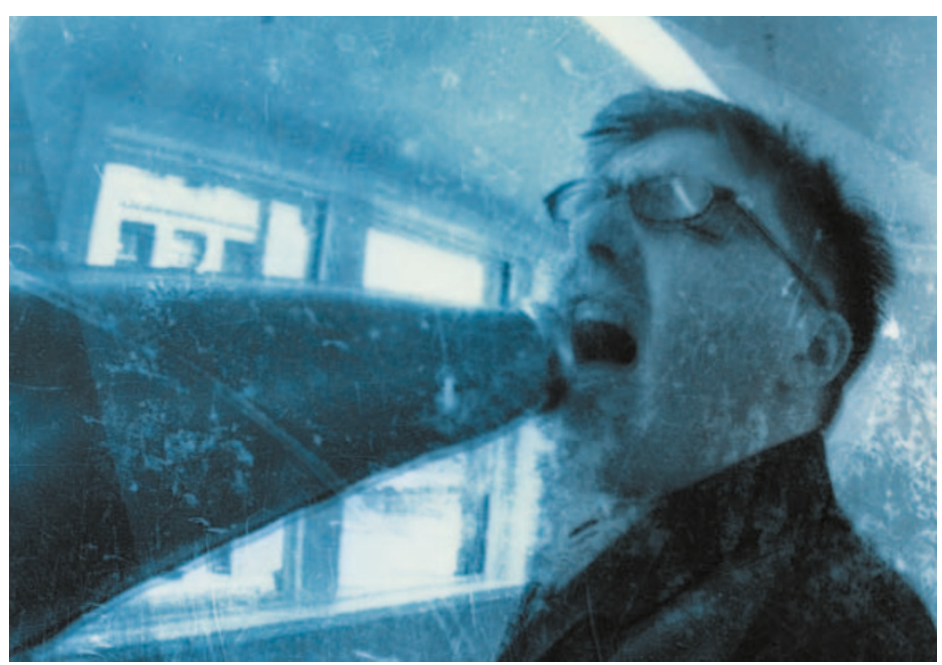

This study identifies RNAi as the Drosophila and mosquito cells, and
Using Drosophila cells transfected with a B2-deficient FHV reporter construct, Li et al. showed that NS1 and E3L - proteins encoded by the mammalian viruses influenza and vaccinia, respectively - were able to suppress antiviral RNA silencing. This activity was seen in three influenza virus genera, indicating it is probably an evolutionarily conserved function of NS1.

The most conserved region of the three NS1 proteins is the dsRNAbinding domain, and mutant NS1 molecules that are unable to bind dsRNA were incapable of inhibiting RNA silencing in Drosophila cells. By contrast, truncated NS1 molecules consisting of the dsRNA-binding domain alone could suppress the RNAi effect, indicating that this domain is both necessary and sufficient for silencing the Drosophila antiviral response. antiviral mechanism of RNA silencing in invertebrate cells. This response is itself suppressed by viral proteins, including some encoded by mammalian viruses, such as influenza and vaccinia, leading the authors to suggest that RNA silencing is an important antiviral response in mammals. Karen Honey

(2) References and links ORIGINAL RESEARCH PAPER Li, W. X. et al. Interferon antagonist proteins of influenza and vaccinia viruses are suppressors of RNA silencing. Proc. Natl Acad. Sci. USA 101, 1350-1355 (2004)

\section{IN BRIEF}

\section{T-CELL SIGNALLING}

\section{Regulation of ZAP-70 activation and TCR signalling by two related proteins, Sts- 1 and Sts-2.}

Carpino, N. et al. Immunity 20, 37-46 (2004)

This paper describes a novel protein — suppressor of T-cell receptor (TCR) signalling 2 (Sts2) - with homology to Sts1. Although Sts2-deficient mice have no haematopoietic lineage defects, in vitro TCR crosslinking led to hyperproliferation of Sts1/Sts2-deficient $\mathrm{T}$ cells, and increased cytokine production. This enhanced responsiveness of Sts1/Sts2-deficient T cells was associated with increased phosphorylation of several TCR-signalling components, including ZAP70, LAT, SLP76 and MAPK, and Sts1/Sts2-deficient mice showed enhanced susceptibility to experimental autoimmune encephalomyelitis. This study establishes a redundant role for Sts1 and Sts2 as negative regulators of TCR signalling, and future work will investigate their mechanism of action.

\section{DENDRITIC CELLS}

IL-10-conditioned dendritic cells, decommissioned for recruitment of adaptive immunity, elicit innate inflammatory gene products in response to danger signals.

Nolan, K. F. et al. J. Immunol. 172, 2201-2209 (2004)

In this study, Nolan et al. identified a novel ligand for CXCchemokine receptor 2 (CXCR2), dendritic cell (DC) inflammatory protein 1 (DCIP1). DCIP1 has homology with mouse CXCchemokine ligand 2 (CXCL2) and, like CXCL2, DCIP1 mRNA was rapidly upregulated after lipopolysaccharide (LPS) stimulation of bone-marrow-derived DCs (BMDCs), indicating a role for DCIP1 early in inflammation. Similar to CXCL2, DCIP1 mediated chemotaxis of a CXCR2-expressing cell line in vitro and neutrophil recruitment in vivo. Treatment of BMDCs with interleukin-10 (IL-10) before LPS exposure enhanced their ability to upregulate the expression of DCIP1 and other pro-inflammatory chemokines, although the induction of chemokines and receptors associated with adaptive immunity was impaired, indicating that IL-10treated BMDCs are a phenotypically distinct BMDC population.

\section{T-CELL ACTIVATION}

Lipid raft proteins have a random distribution during localized activation of the T-cell receptor.

Glebov, O. O. \& Nichols, B. J. Nature Cell Biol. 3, 238-243 (2004)

Fluorescence resonance energy transfer (FRET), which can detect small clusters of molecules, was used to study the role of lipid-raft microdomains in T-cell synapse formation. Are components recruited through protein-protein interactions, or are they clustered in lipid rafts, which redistribute to the synapse? By expressing glycophosphatidylinositol (GPI)-linked FRET donor and acceptor proteins in Jurkat $\mathrm{T}$ cells, the authors showed that the distribution of lipid rafts (identified by the GPI-linked proteins) throughout the cell membrane was not altered by activation with CD3-specific antibody. It will now be necessary to determine whether physiological T-cell synapses also form without requiring lipid-raft redistribution. 